\title{
Fahr's Disease Presenting as Bipolar Affective Disorder - Case Report
}

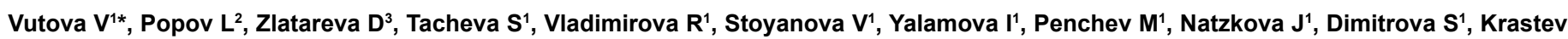
$\mathrm{St}^{1}$ and Milanova $\mathrm{V}^{1}$

${ }^{1}$ Psychiatric Clinic, University Hospital "Alexandrovska", Sofia, Bulgaria

${ }^{2}$ Clinic of Neurology, University Hospital "Alexandrovska", Sofia, Bulgaria

${ }^{3}$ Clinic of Diagnostic Imaging, University Hospital "Alexandrovska", Sofia, Bulgaria

\begin{abstract}
We present a case of a 59-year-old woman with bipolar affective disorder and idiopathic calcium accumulation in the basal ganglia (lenticular nuclei). Her physical and neurological examinations were without clinically significant abnormalities. Neuropsychological assessment shows mild cognitive deficit. Clinical and laboratory data meet the diagnostic criteria for Fahr's disease. Discussions were held about the relationship between calcification of basal ganglia and the occurrence of the bipolar disorder.
\end{abstract}

Keywords: Fahr's disease; Basal ganglia; Calcifications; Bipolar affective disorder

\section{Introduction}

Basal ganglia play an important role both in the direct realization of the motor function but also in the control of motor behavior on the motivation level [1-10].

Fahr's disease is a rare degenerative disorder, characterized by an idiopathic bilateral and symmetrical accumulation of calcium in the different structures of the basal ganglia, most frequently in the striopallidar region (Figure 1). The disease was first described by the German neurologist Fahr in 1930. The secondary calcium accumulation in the basal ganglia (e.g. in endocrine-metabolic pathway), is defined as a Fahr's syndrome [7-12].

The disease manifests itself clinically in various neurological, psychiatric and cognitive disturbances. It is most often observed as movement disorders like parkinsonian or choreoathetosis syndrome. Sometimes coordination and language symptoms are observed and rarely any epileptic phenomena $[7,12]$. The psychiatric features more often include a demented or depressive syndrome, but there are also psychotic, affective, anxiety and personality disorders $[3,6,8,9,12]$ The disease usually starts between the ages 40 and 60 with a gradual course and progressive deterioration. It is believed that asymptomatic forms can also exist [7].

We report a case of bipolar affective disorder with late onset and a progressively deteriorating course in which idiopathic bilateral and symmetrical accumulation of calcium in the basal ganglia (lenticular nuclei) was established.

\section{Case Report}

The patient is a 59-year-old woman with secondary education, married, with no evidence of stressogenic psychosocial factors.

She has a patrilineal family history with affective disorders (aunt, two cousins and nephew). No significant past diseases and comorbidities.

The psychiatric disorder started in 2010 (53 years old) with manifestations of depressive symptoms that resolved spontaneously. An identical status occurred in 2011. In January 2013 a third depressive episode occurred and was resolved with an outpatient antidepressant treatment. From January 2014 annual depressive episodes followed, including depressive-psychotic features and the more rare, but also annual moderate manic episodes. The disease acquires a bipolar course with a tendency toward a progressive deterioration of the frequency and severity of the relapses. The patient underwent a regular maintenance therapy. Complete, but short remissions were observed.

In January 2016 the woman was hospitalized in Psychiatric Clinic of UMHAT "Alexandrovska"-Sofia because of a recent manic deterioration. Upon registering at the hospital no abnormalities were found during the physical examination except data for varices in the right leg and slight impermanent tremor of the hands. The mental status revealed a classic manic state without psychotic features. The main manic symptoms were elevated mood, over activity, inflated selfesteem, increase talkativeness and mild decreased need for sleep.

12 days later, simultaneously with manic behavior, short-term, non congruent, simple visual hallucinations occurred. They were established every day in clear consciousness and the patient has been critical to them.

The standard laboratory tests, including TSH and FT4, alkaline phosphatase, calcium, phosphate, parathyroid hormone have shown no clinically significant abnormalities. Neuropsychological assessments have indicated to discrete signs of organic damage and early cognitive deficits (MMSE - p. 27). The examination made by a neurologist and an endocrinologist found no evidence of neurological or endocrinological disorders. Computer Tomography (CT) and Magnetic Resonance Imaging (MRI) of the brain showed focal calcifications in the basal ganglia involving lenticular nuclei only (Figures 2-4).

Depakine chrono (Sodium valproate) was administered to the patient with - up to $1500 \mathrm{mg} / \mathrm{d}$. (pl. level of up to $630 \mathrm{mcmol} / \mathrm{l}$ ), Olanzapine - up to $10 \mathrm{mg} / \mathrm{d}$., Lithium carbonate - up to $600 \mathrm{mg} / \mathrm{d}$. (pl. level of $0.57 \mathrm{mcmol} / \mathrm{l})$, Chlorprothixene - up to $30 \mathrm{mg} / \mathrm{d}$., Ucerax - up to $75 \mathrm{mg} / \mathrm{d}$., Nootropil -3 g. i.v.

In the course of the treatment the patient showed gradual but

*Corresponding author: Vutova V, Psychiatric Clinic, University Hospital "Alexandrovska", Sofia, Bulgaria, Tel: 3598986141 65; E-mail: vasyavutova@grnail.com

Received April 29, 2017; Accepted May 05, 2017; Published May 15, 2017

Citation: Vutova V, Popov L, Zlatareva D, Tacheva S, Vladimirova R, et al. (2017) Fahr's Disease Presenting as Bipolar Affective Disorder - Case Report. J Ment Disord Treat 3: 135. doi:10.4172/2471-271X.1000135

Copyright: ( 2017 Vutova V, et al. This is an open-access article distributed unde the terms of the Creative Commons Attribution License, which permits unrestricted use, distribution, and reproduction in any medium, provided the original author and source are credited. 
Citation: Vutova V, Popov L, Zlatareva D, Tacheva S, Vladimirova R, et al. (2017) Fahr's Disease Presenting as Bipolar Affective Disorder - Case Report. J Ment Disord Treat 3: 135. doi:10.4172/2471-271X.1000135

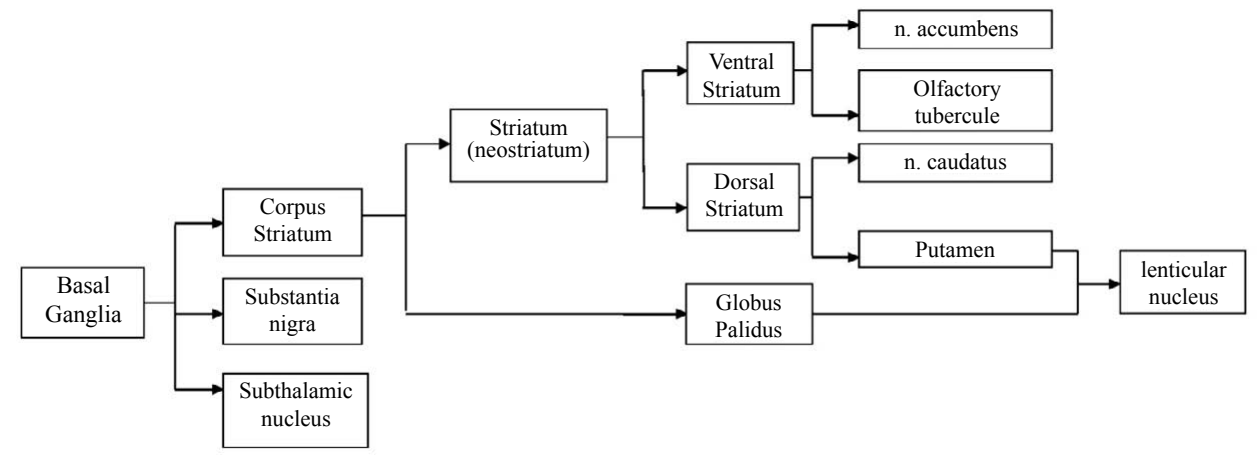

Figure 1: Structure of the basal ganglia.

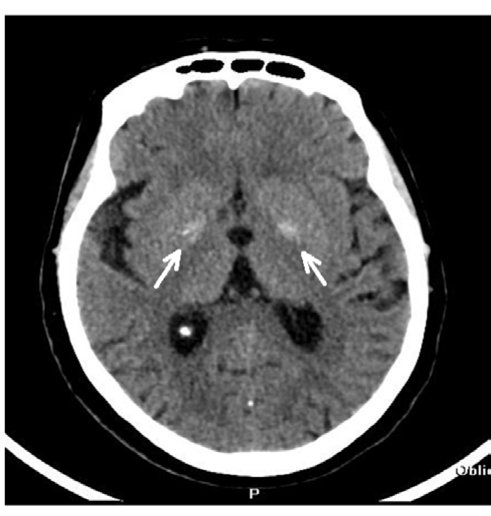

Figure 2: CT of the brain (axial image). In lenticular nuclei were visualized bilaterally and show hyperdense.

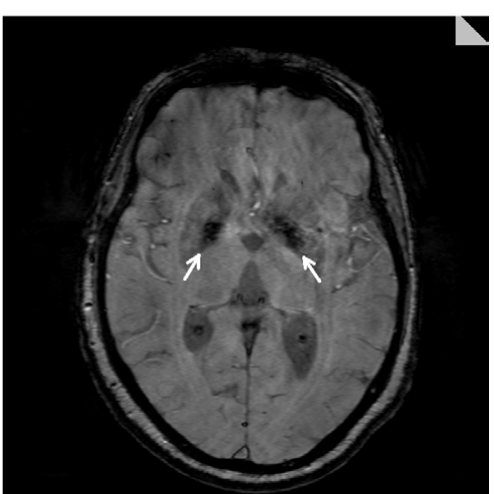

Figure 3: MRI of the brain (axial image, SWI). The lenticular nuclei were visualized bilaterally and show zones with no signals representing calcification (see arrows).

marked improvement. Hallucinations stopped within 10 days. The manic symptoms reduced significantly. The patient was discharged in complete remission in February 2016. It was recommended to continue the treatment with Depakine chrono - $1000 \mathrm{mg} / \mathrm{d}$. (pl. level of 457 $\mathrm{mcmol} / \mathrm{l}$,) Lithium carbonate $-600 \mathrm{mg} / \mathrm{d}$. (pl. level $-0.57 \mathrm{mcmol} / \mathrm{l}$ ), and Nootropil - $2400 \mathrm{mg} / \mathrm{d}$.

\section{Discussion}

Fahr's disease is a neurodegenerative disorder associated with idiopathic bilateral calcium accumulation in the basal ganglia. It is assumed that a genetic mechanism lays at the basis of the etiology. It

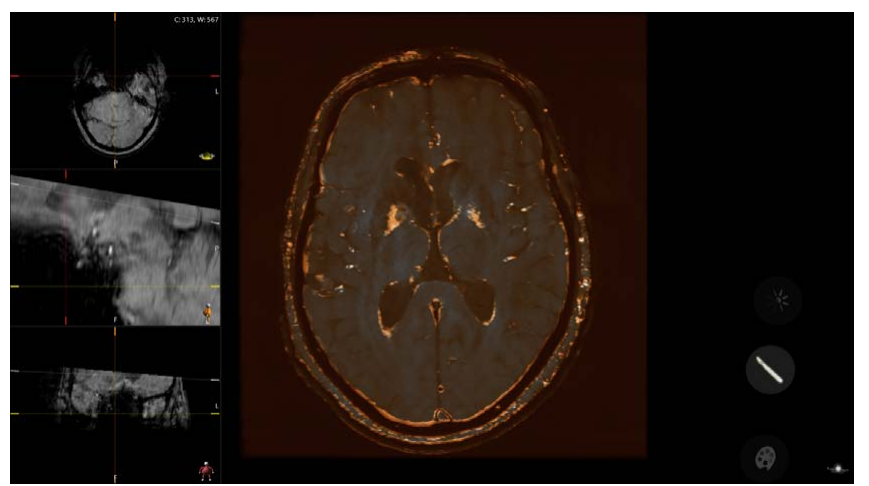

Figure 4: The same axial image presented by 3D virtual dissection table.

is inherited autosomally, but sporadic cases have also been observed. Several genes have an impact with a predominantly role of IBGC1 locus of chromosome $14 \mathrm{q}$ as well as the heterozygous mutation in the SLC20A2 locus of chromosome 8r11 [5,11].

The pathogenesis is characterized by striopallidar nonatherosclerotic bilateral and symmetric calcifications. Hypometabolism of the basal ganglia and frontal lobe of the brain has been reported. It is assumed a disruption of cortical-subcortical connections in normal nigrostriatal pathway [2,7].

The idiopathic calcification of basal ganglia shows a wide range of clinical manifestations which depends on the degree of the damage and the location of the affected structures. The clinical presentation includes combined neurological and psychiatric symptoms, neurological symptoms only and, less frequently, psychiatric symptoms only $[3,6,7,9,12]$. In symptomatic patients calcification is more massive compared to the asymptomatic ones [7].

The differential diagnosis is based primarily on parathyroid imbalance, vascular encephalopathy, trauma and other disorders leading to calcium deposits.

Psychiatric features in Fahr's disease are initial symptoms in about $40 \%$ of the cases [7]. However, the pathophysiological basis for their occurrence remains unclear $[4,6]$. More frequent observations include depression, psychotic and cognitive disorders $[3,8,12]$. The presented case is that of a 59-year-old woman with a bipolar affective disorder with a progressively deteriorating course of severity and frequency of relapses without significant neurologic or somatic symptoms. During the recent relapse, in addition to the classic manic symptoms, 
Citation: Vutova V, Popov L, Zlatareva D, Tacheva S, Vladimirova R, et al. (2017) Fahr's Disease Presenting as Bipolar Affective Disorder - Case Report. J Ment Disord Treat 3: 135. doi:10.4172/2471-271X.1000135

Page 3 of 3

organic-type psychotic symptoms were also found. This requires extensive studies to show idiopathic symmetric and mild accumulation of calcium in the basal ganglia (lenticular nuclei), as well as discrete cognitive deficits. Clinical analysis indicate that at this stage there are major diagnostic criteria for sporadic cases of Fahr's disease, namely: onset between 40 and 60 years of age, bilateral calcification of basal ganglia, progressive neuropsychiatric disfunction (bipolar disorder with deteriorating course and initial cognitive deficits), absence of other causes, initiating such disturbances.

We explain the absence of neurological symptoms with the possibility that the disease may be in its early stages as well as with the fact that calcification is mild and has a specific location with an isolated involvement of lenticular nuclei. For the final diagnosing long-term monitoring of the patient will be necessary because of the possibility that the bipolar disorder and calcifications of the basal ganglia may be a manifestation of comorbidity with no correlation between them.

\section{Conclusion}

Impaired functions of the basal ganglia lead to various neurological and psychiatric diseases. Fahr's disease is a rare neurodegenerative disorder with high genotypic and phenotypic heterogeneity. Further research is needed to determine the pathophysiological role of idiopathic calcifications of the basal ganglia in the psychiatric syndromes. The presence of a bipolar disorder in a case of isolated damage of the basal ganglia may be taken into account in the study of the organic base of affective disorders. We believe that it is appropriate idiopathic calcification of basal ganglia which has to be considered in the differential diagnosis of psychiatric syndromes.

\section{References}

1. Milanov K, Milanova V (2003) Mind's formations. B: Biological Psychiatry Schizophrenia and Antipsychotics. Boyko Stamenov Company, Sofia.

2. Benke T, Karner E, Seppi K, Delazer M, Marksteiner J, et al. (2004) Sub acute dementia and imaging correlates in a case of Fahr's disease. J Neurol Neurosurg Psychiatry 75: 1163-1165.

3. Ghormode D, Maheshwari U, Kate N, Grover S (2011) Fahr's disease and psychiatric syndromes: A case series. Ind Psychiatry J 20: 136-138.

4. Hibar DP, Westyle LT, Erp TGM (2016) Subcortical volumetric abnormalities in bipolar disorder. Molecular Psychiatry 227: 1-7.

5. Hsu SC, Sears RL, Lemos (2013) Mutations in SLC20A2 are a major cause of familial idiopathic basal ganglia calcification. Neurogenetics 14: 11-22.

6. Konig P (1989) Psychopathological alterations in cases of symmetrical basal ganglia sclerosis. Biol Psychiatry 25: 459-468.

7. Amir A, Al-Hassan GA (2014) Familial idiopathic basal ganglia calcification (Fahr's disease). Neurosciences $19: 171-175$.

8. Shakibai SV, Johnson JP, Bourgeois JA (2005) Paranoid delusions and cognitive impairment suggesting Fahr's disease. Psychosomatics 46: 569-572.

9. Shoib S, Dar MM, Arif T, Bashir H, Ahmed J (2012) Fahr's disease presenting as mania: A case report. Iran J Psychiatry Behav. Sci 6: 102-104.

10. Schultz W, Tremblay L, Hollerman JR (1998) Reward prediction in primate basal ganglia and frontal cortex. Neuropharmacology 37: 421-429.

11. Yamada M, Tanaka M, Takagi (2014) Evaluation of SLC20A2 mutations that cause idiopathic basal ganglia calcification in Japan. Neurology 82: 705-712.

12. Wirshing CW (1995) Neuropsychiatric aspects of movement disorders In: Kaplan HI, Sadock BJ, Baltimore (eds.) Comprehensive Handbook of Psychiatry, Williams and Wilkins, 1: 220-231. 